\title{
Brain Activation during Input from Mechanoinsensitive versus Polymodal C-Nociceptors
}

\author{
Belinda Susanne Ruehle, Hermann Otto Handwerker, Jochen Klaus Maria Lennerz, Ralf Ringler, and Clemens Forster \\ Institute of Physiology and Pathophysiology, University of Erlangen/Nuernberg, D-91054 Erlangen, Germany
}

C-nociceptors mediating cutaneous pain in humans can be distinguished in mechano-heat-responsive units (CMH) and mechanoinsensitive units $\left(\mathrm{CM}_{\mathrm{i}}\right)$. However, if sensitized in damaged tissue, $\mathrm{CM}_{\mathrm{i}}$ play an important role in inflammatory pain. $\mathrm{CM}_{\mathrm{i}}$ differ from $\mathrm{CMH}$ by higher electrical thresholds and by mediating the axon reflex. Using these properties, we established two stimulation paradigms: (1) transcutaneous stimulation (TCS) of low current density below the $\mathrm{CM}_{\mathrm{i}}$ threshold and (2) intracutaneous stimulation (ICS) of high current density that excites $\mathrm{CM}_{\mathrm{i}}$. This was proven by the quantification of the axon-reflex flare. Applying these stimulation paradigms during functional magnetic resonance imaging, we investigated whether nociceptor stimulation that recruits $\mathrm{CM}_{\mathrm{i}}$ leads to different cerebral activation than stimuli that do not recruit $\mathrm{CM}_{\mathrm{i}}$. Brain activation by $\mathrm{CM}_{\mathrm{i}}$ was inferred by subtraction. Both stimuli recruited multiple afferents other than $\mathrm{CM}_{\mathrm{i}}$, and we expected a common network of regions involved in different aspects of pain perception and motor nocifensive reactions in both stimuli. ICS that additionally recruited $\mathrm{CM}_{\mathrm{i}}$ should activate regions with low acuity that are involved in pain memory and emotional attribution. Besides a common network of pain in both stimuli, TCS activated the supplementary motor area, motor thalamic nuclei, the ipsilateral insula, and the medial cingulate cortex. These regions contribute to a pain processing loop that coordinates the nocifensive motor reaction. $\mathrm{CM}_{\mathrm{i}}$ nociceptor activation did not cause relevant activation in this loop and does not seem to play a role in withdrawal. The posterior cingulate cortex was selectively activated by ICS and is apparently important for the processing of inflammatory pain.

Key words: pain; nociception; electric stimulation; fMRI; imaging; cortical; brain

\section{Introduction}

It has been known since Erlanger and Gasser that nociceptors comprise thin and slowly conducting nerve fibers (Gasser, 1935). Most of them are unmyelinated C-fibers, and recently a subclass with peculiar properties has been described in human skin (Schmidt et al., 1995; Schmelz et al., 1997). These nociceptors are unresponsive to mechanical stimulation, including pinprick as long as the surrounding tissue is intact. They become mechano responsive in the course of inflammation (Schmelz et al., 1994). This behavior earned them the poetic label "sleeping nociceptors" (Schaible and Schmidt, 1988). First described in the monkey (Meyer and Campbell, 1981; Garell et al., 1996), these cutaneous mechano-insensitive $\mathrm{C}$-nociceptors $\left(\mathrm{CM}_{\mathrm{i}}\right)$ comprise $\sim 30 \%$ of afferent C-units in human skin (Schmidt et al., 1995) and are different from polymodal mechano-heat-sensitive C-nociceptors $(\mathrm{CMH})$ in many respects. $\mathrm{CM}_{\mathrm{i}}$ have larger receptive fields (Schmidt et al., 2002) and higher heat thresholds (Weidner et al., 1999). Some are particularly sensitive to histamine and mediate itch sensation (Schmelz et al., 1997). $\mathrm{CM}_{\mathrm{i}}$ are

\footnotetext{
Received May 21, 2005; revised April 10, 2006; accepted April 10, 2006.

This work was supported by the Deutsche Forschungsgemeinschaft. We gratefully acknowledged the help of $R$. Carr and K. Zimmermann in the preparation of this manuscript.

Correspondence should be addressed to Dr. Clemens Forster, Institute of Physiology and Pathophysiology, University of Erlangen/Nuernberg, Universitaetsstrasse 17, D-91054 Erlangen, Germany. E-mail: forster@ physiologie1.uni-erlangen.de.

DOI:10.1523/JNEUROSCI.2059-05.2006

Copyright $\odot 2006$ Society for Neuroscience $\quad$ 0270-6474/06/265492-08\$15.00/0
}

more sensitive to stimulation by inflammatory mediators than $\mathrm{CMH}$ (Schmelz et al., 2000). Given these characteristics, it seems likely that $\mathrm{CM}_{\mathrm{i}}$ are rather involved in hyperalgesia and inflammatory pain than in acute nocifensive reactions (Schmidt et al., 2000). Interestingly, thresholds for transcutaneous electrical stimulation are significantly higher in $\mathrm{CM}_{\mathrm{i}}$ (Weidner et al., 1999; Schmidt et al., 2002). Based on this knowledge, Schmelz et al. (2000) demonstrated that an axon-reflex flare (Lynn et al., 1996) is only elicited in human skin when the stimulus strength is high enough to recruit $\mathrm{CM}_{\mathrm{i}}$.

The clearly separated threshold distribution opens experimental approaches to study the role of $\mathrm{CM}_{\mathrm{i}}$. Electrical stimuli can be chosen to excite receptor populations including or excluding $\mathrm{CM}_{\mathrm{i}}$. We will show here that the critical parameter is not voltage but current density at the nociceptor terminals.

To gain insight into cerebral processing of $\mathrm{CM}_{\mathrm{i}}$, we established two stimulation paradigms: (1) transcutaneous stimulation (TCS) of low current density below the $\mathrm{CM}_{\mathrm{i}}$ threshold and (2) intracutaneous stimulation (ICS) of high current density that excites $\mathrm{CM}_{\mathrm{i}}$, evidenced by quantification of the axon-reflex flare.

Although the central mechanisms of hyperalgesia have been studied previously (Mailhoefner et al., 2004; Mailhoefner and Handwerker, 2005), $\mathrm{CM}_{\mathrm{i}}$ contribution to the emerging cerebral activation pattern is unclear. We investigated activation patterns of ICS (including $\mathrm{CM}_{\mathrm{i}}$ ) versus TCS (excluding $\mathrm{CM}_{\mathrm{i}}$ ) by functional magnetic resonance imaging (fMRI). Subsequent subtraction provides indirect evidence of the cerebral correlates of $\mathrm{CM}_{\mathrm{i}}$ 
input. Zambreanu et al. (2005) recently suggested a role of the brainstem, cerebellum, and other subcortical and limbic areas in hyperalgesia. We focused on these areas.

According to $\mathrm{CM}_{\mathrm{i}}$ function, brain areas activated by ICS should play a role in hyperalgesia and inflammatory pain. We expected these to be regions with low acuity such as the cingulate cortex and limbic areas involved in pain memory and emotional attribution (Bromm, 2001; Vogt, 2005). Because both stimuli excite "non- $\mathrm{CM}_{\mathrm{i}}$ " fibers (e.g., $\mathrm{CMH}$ and $\mathrm{A} \delta$ ) in different quantities (TCS > ICS), TCS and ICS should activate regions with high acuity for nocifensive reactions (e.g., motor integrative and cerebellar areas).

\section{Materials and Methods}

General. Thirteen healthy subjects, age 20-30 years, participated in each the psychophysical/laser-Doppler experiments and in the fMRI study. Nine of them were female. Four subjects, three females, participated in both studies. Subjects gave their informed consent and were instructed that they could withdraw from the experiment at any time. The study was approved by the local ethics committee.

Before the experiment, subjects became familiar with the stimulation procedure and with the experimental protocol. The studies were single blind. Individual sensation and pain thresholds were determined before every experimental session. To determine pain quality, we asked subjects to describe the sensation in their own words. Thresholds were acquired by gradually increasing the current and were analyzed for differences in magnitude and variance between the different experiments and between genders by $F$ and $t$ tests. Data on the female cycle were not collected.

Stimulus design and application. Electrical stimuli (width, $0.5 \mathrm{~ms}$; frequency, $1 \mathrm{~Hz}$ ) were applied from a constant current source (Type DS7A, serial D127A; Digitimer, Welwyn Garden City, UK) for 20 s periods. For TCS, two epicutaneous ECG electrodes $(17 \mathrm{~mm}$; ARBO; Adshead Ratcliffe \& Company, Derbyshire, UK) were attached $1.5 \mathrm{~cm}$ apart to the dorsum of the foot. For ICS, two hypodermic needles $(0.5 \times 25 \mathrm{~mm}$; Microlance, number 18; Becton Dickinson, Mountain View, CA) were inserted intracutaneously $1.5 \mathrm{~mm}$ apart and each to a depth of $2 \mathrm{~mm}$.

Psychophysics and laser Doppler imaging. Intracutaneous needle electrodes were inserted in the right $(n=6)$ or left $(n=5)$ dorsum of the foot. Epicutaneous electrodes were attached to the opposite foot. A $30 \mathrm{~min}$ period was allowed between needle insertion and stimulation to let the flare by needle insertion completely fade. Subjects were instructed to rate each stimulation on a $0-10$ numeric pain rating scale. TCS started with 5 $\mathrm{mA}$. The current was increased stepwise $(5,10,15,20,25,30,40,50,60$, and $70 \mathrm{~mA}$ ) until the pain was rated 7 on a scale from 1 to 10 or until the ceiling of $70 \mathrm{~mA}$ was reached. ICS started with $1 \mathrm{~mA}(1,2,4,6,8,10,13$, 16,20 , and $24 \mathrm{~mA}$ ). The cutoff was either a pain rating of 7 or the ceiling $24 \mathrm{~mA}$. Subjects who reported pain levels $<7$ at the maximal stimulation intensity were excluded from the study.

Blood flux measurements were performed using a Laser Doppler Imager (Moor Instruments, Wilmington, DE) positioned over the dorsum of the foot. The scanned field (size, $4.5 \mathrm{~cm}^{2}$; scan time for each image, $30 \mathrm{~s}$ ) completely covered the stimulation area. After recording a $30 \mathrm{~s}$ baseline (first image), stimulation was applied during the first $20 \mathrm{~s}$ of the second image. Subsequently, an additional seven $30 \mathrm{~s}$ images were obtained. For each stimulus level, a series of nine pictures (4.5 $\mathrm{min}$ ) was recorded.

Laser Doppler imaging (LDI) software (LDI version 3.08; Moor Instruments) was used for evaluation. The baseline was subtracted from all poststimulus scans. The average flux increase was determined in a region of interest (ROI) (see Fig. 2). For ICS, the ROI was chosen at a distance from the needles to avoid the erythema caused by needle insertion. Flux increase and flux variance in the two genders were analyzed by the $F$ test and $t$ test to detect intersexual differences.

fMRI of the brain. Epicutaneous electrodes for TCS were attached to the dorsum of the right foot. Intracutaneous needle electrodes for ICS were positioned between the epicutaneous electrodes. Although the stimulation area in TCS was larger, this positioning granted topographically identical stimulation sites. Stimulation strengths were determined

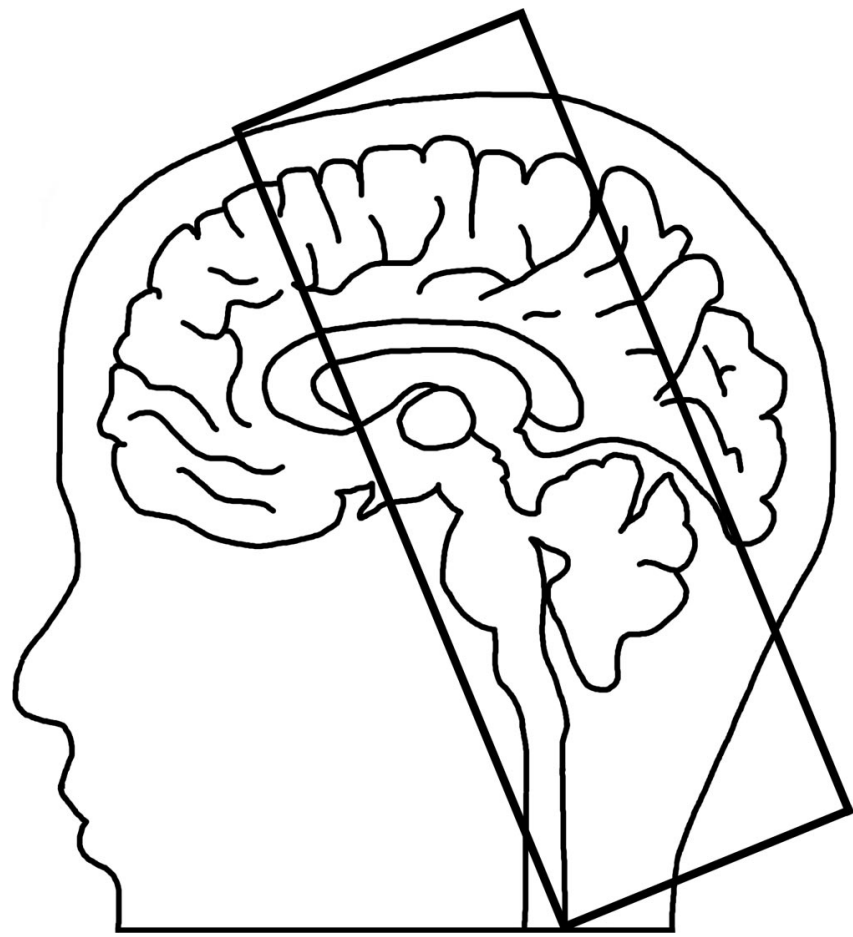

Figure 1. Positioning of the EPI slices for the collection of the functional data. The block consisted of 16 slices, each with a thickness of $4 \mathrm{~mm}$. The scan time was a repetition time of $2.5 \mathrm{~s}$ per block.

before each session to obtain a pain rating of 7. Each fMRI session consisted of two separate trials for either TCS or ICS. In each trial, five $20 \mathrm{~s}$ stimuli of the same type (either ICS or TCS) were applied. The baseline time between stimuli was varied to avoid bias by pain anticipation (Ploghaus et al., 1999). The fMRI session was started randomly with the TCS trial in nine cases. To avoid task-related bias, we did not obtain a simultaneous rating. Instead, the same protocol was applied in a separate session without imaging to all subjects, and an on-line rating through the five stimulation periods was recorded with a visual rating scale.

Imaging was performed using a $1.5 \mathrm{~T}$ Sonata MRI scanner (Siemens, Erlangen, Germany). For each subject, a T1-weighted magnetizationprepared rapid gradient echo data set was recorded with a high threedimensional resolution covering the entire brain (160 sagittal slices; thickness, $1.5 \mathrm{~mm}$; in-plane resolution, $0.98 \times 0.98 \mathrm{~mm}^{2}$; field of view, $250 \times 250 \mathrm{~mm}^{2}$; data matrix, $\left.256 \times 256\right)$. Functional data were collected using a multislice echo planar imaging (EPI) technique. Each functional data set consisted of 93 blocks, visualizing 16 slices (Fig. 1) [repetition time, $114 \mathrm{~ms}$; echo time, $62 \mathrm{~ms}$; flip angle, $90^{\circ}$; scan time, $2.5 \mathrm{~s}$ per block of 16 slices; slice thickness, $4 \mathrm{~mm}$; field of view, $220 \times 220 \mathrm{~mm}^{2}$; data matrix, $128 \times 128$ ] (Ringler et al., 2003). Stimulation was administered during blocks $14-20,29-35,46-52,60-66$, and 77-83.

The field of view was chosen to completely cover the thalamus, brainstem, cerebellum, and the following cortical areas often described to be involved in the pain network: supplementary motor area (SMA), secondary motor cortex (S2), medial and posterior insular cortices, and medial and posterior cingulate cortices. As a consequence of this selection, however, S1, the anterior pole of the insula, and the perigenuate cingulate cortex were outside of this block.

fMRI data were statistically analyzed with BrainVoyager software version 4.8 (Brain Innovations, Maastricht, The Netherlands). Functional and anatomical images of all subjects were superimposed and transformed into Talairach space (Talairach and Tournoux, 1988). The threedimensional preprocessing of the functional data included head movement assessment, slice scan time correction, high-frequency filtering, and removal of linear trends. Changes in the blood oxygenation level (BOLD) signal were statistically evaluated using a general linear model (GLM) with the stimulus function as a predictor that corresponded to a 
boxcar design. Data were low-pass filtered (cutoff frequency, $0.2 \mathrm{~Hz}$ ) to account for the hemodynamic response delay (Boynton et al., 1996). A minimal cluster size of 80 pixels was used as a cutoff to eliminate very small or random activations. Clusters were assigned manually to anatomical loci by a professional viewer based on a printed brain atlas (Kretschmann and Weinrich, 1992; Damasio, 1995). The $t$ values obtained from the GLM analysis were used to compare activation patterns. $p$ values $<0.05$ were regarded as significant. Findings were verified by processing the centric coordinates with the Talairach Daemon Client internet software (http://ric.uthscsa.edu/projects/talairachdaemon. html). Group analyses, which included the data of all subjects during either type of stimulus, were performed separately on each of the two trials. A third group analysis was performed for contrast evaluation. It was done with the $z$ transformed data of both trials, and the predictors were stimulus onset and type of stimulus. To characterize the most relevant activated regions, two parameters reflecting the difference between baseline and fMRI signal during the stimuli were selected: BOLD contrast and $t$ value. For each brain ROI, the number of subjects showing significant activation was listed. Data of male versus female subjects were analyzed in a set of two Multi Studies to detect gender differences.

\section{Results}

\section{Pain perception}

ICS-induced pain was uniformly described as "stinging," whereas TCSinduced pain was perceived as "deep," "pounding," or "dull." Because these descriptions were given in an unstructured form, no statistics can be provided. However, no subject described ICS as deep, pounding, or dull or described TCS as stinging. Four of 17 subjects felt TCS to be more unpleasant, 5 subjects felt ICS to be more unpleasant, whereas 8 subjects found both types of stimulation likewise unpleasant. Pain thresholds varied widely. During the LDI experiments, TCS became painful at $18.9 \mathrm{~mA}$, on average, (range, 11.1-29.1). Pain ratings increased to 7 when the current was raised to $40.8 \mathrm{~mA}$, on average (range, 20.0-68.3). TCS thresholds during the fMRI session were lower at $10.7 \mathrm{~mA}$, on average (range, 4.0-20.6), and the current required to reach a rating of 7 was $30.3 \mathrm{~mA}$, on average (range, 15.0-44.1). For ICS, thresholds and rating 7 currents were equal in both experiments. The mean pain threshold was 2.7 $\mathrm{mA}$ (range, 0.2-6.0), and rating 7 was reached at $14.9 \mathrm{~mA}$, on average (range, 6.3-49.3). There was no significant difference in pain perception regarding quality, threshold variance ( $F$ test), or threshold ( $t$ test) between the two genders. A simultaneous rating over the five stimulation periods of the fMRI stimulation protocol showed a consistent rating of 7 during all five stimulation periods and a complete cessation of pain during baseline periods.

\section{LDI: local stimulation effects}

In the LDI experiments, one subject had to be excluded because a pain rating of 7 was not reached at the maximal stimulation intensity of $24 \mathrm{~mA}$ for ICS. Two subjects were excluded for movement artifacts. Consequently, the data from 10 subjects were analyzed. No erythema was observed even at the highest TCS b)

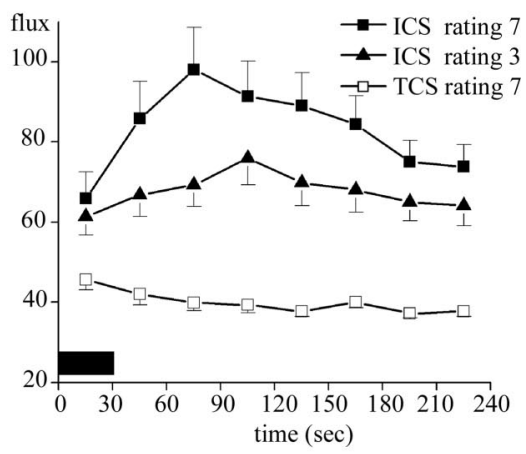

c)

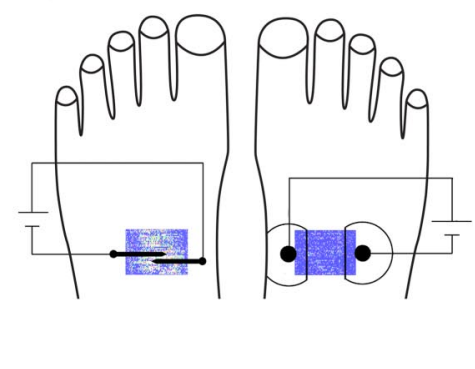

Figure 2. Flare reactions in the skin of the dorsum of the foot. $\boldsymbol{a}$, Baseline-subtracted LDIs from one representative subject for (The ROI and electrode positions are depicted in the first image (baseline). A stimulus that lasted 20 s (ICS, 8 mA; TCS, stimulation is covered. Flux values are encoded by the pixel color. The corresponding flux values are displayed in the scale on the 作 incease at any level of stimulation. Thus, only flux at rating 7 is displayed. $c$, Map of the electrode positions for the LDI trial: needle electrodes for ICS are exemplary displayed on the dorsum of the left foot, and surface electrodes for TCS exemplary displayed on the dorsum of the right foot. Scanned fields are plotted in blue. These scanned fields correspond to the displayed area in $\boldsymbol{a}$.

stimulus levels (70 mA), and no subject showed TCS-induced flux increases in LDI. Before ICS measurements were started, the erythema caused by the insertion of the needles had completely faded in all subjects. Baseline LDI measurements still showed an increased flux and were subtracted. After ICS stimulation, a visible erythema developed again. This was especially pronounced at higher stimulus intensities. The LDI flux values clearly rose after the stimulus and reached a maximum $\sim 90 \mathrm{~s}$ after stimulation. Maximal and integrated flux was positively correlated to stimulation intensity for ICS (Fig. 2), resulting in a maximal difference between ICS and TCS flux values at rating 7. Integrated and maximal flux after ICS was greater than after TCS in every single subject (integrated flux after baseline subtraction at rating 7: ICS: mean, 576.5; SE 58.0; TCS: mean, 274.4; SE, 10.1.) Integrated flux ( $t$ test) and variance ( $F$ test) were not significantly different between the two genders.

\section{Cerebral responses: BOLD effects}

Two subjects were excluded because of movement artifacts, and accordingly, the data from 11 subjects were analyzed. Table 1 lists activations in the most relevant ROIs during ICS and TCS stimulation. In addition, we found that motor, visual, and auditory cortical areas were activated in single subjects. Because these activations occurred only in single subjects, they were not included in the tables. A group analysis using the predictor gender showed 
Table 1. Stimulus-dependent activation in the brain ROIs

\begin{tabular}{|c|c|c|c|c|c|c|c|c|c|c|c|c|c|c|}
\hline \multirow[b]{2}{*}{ Region } & \multicolumn{7}{|c|}{ TCS } & \multicolumn{7}{|l|}{ ICS } \\
\hline & $n$ & Voxel & BOLD & $t$ & $x$ & $y$ & $z$ & $n$ & Voxel & BOLD & $t$ & $x$ & $y$ & $z$ \\
\hline SMAI & 6 & 2489 & 0.24 & 6.68 & -1 & -4 & 49 & 7 & & & & & & \\
\hline SMA r & 4 & 1462 & 0.17 & 6.54 & 4 & -2 & 45 & 5 & & & & & & \\
\hline S2I & 9 & 4910 & 0.26 & 7.85 & -54 & -15 & 18 & 6 & 4629 & 0.15 & 3.90 & -55 & -13 & 16 \\
\hline$S 2 r$ & 8 & 3124 & 0.24 & 8.52 & 55 & -25 & 23 & 6 & 1937 & 0.14 & 6.02 & 59 & -22 & 23 \\
\hline MICI & 8 & 4626 & 0.23 & 3.20 & -41 & -3 & 12 & 6 & 3930 & 0.07 & 6.42 & -35 & -10 & 9 \\
\hline $\mathrm{MIC} r$ & 7 & 6671 & 0.85 & 4.43 & 38 & 2 & 14 & 9 & & & & & & \\
\hline PICI & 6 & 4902 & 0.16 & 8.97 & -42 & -27 & 20 & 5 & 2056 & 0.04 & 6.27 & -37 & -22 & 16 \\
\hline $\mathrm{MCCl}$ & 10 & 5042 & 0.31 & 5.83 & -5 & 0 & 37 & 10 & 1677 & 0.05 & 5.27 & -6 & -8 & 41 \\
\hline $\mathrm{MCC} r$ & 10 & 1946 & 0.35 & 3.58 & 5 & 3 & 35 & 10 & & & & & & \\
\hline PCCI & 2 & & & & & & & 5 & 217 & 0.13 & 4.40 & 0 & -26 & 26 \\
\hline $\mathrm{PCC} r$ & 2 & & & & & & & 5 & 225 & 0.06 & 4.60 & 4 & -26 & 27 \\
\hline Inferior parietal lobe $r$ & 6 & 3655 & 0.30 & 7.86 & 54 & -27 & 32 & 6 & 3496 & 0.24 & 7.01 & 53 & -26 & 25 \\
\hline Inferior parietal lobe I & 6 & 3438 & 0.24 & 8.00 & -52 & -29 & 29 & 7 & 1863 & 0.23 & 6.28 & -53 & -25 & 27 \\
\hline Thalamus | & 6 & 1015 & 0.12 & 6.23 & -14 & -14 & 5 & 9 & 982 & 0.09 & 5.22 & -10 & -19 & 4 \\
\hline Thalamus r & 6 & 323 & 0.08 & 6.34 & 6 & -12 & -3 & 5 & 643 & 0.08 & 5.17 & 5 & -15 & 2 \\
\hline Brainstem I & 5 & 618 & 0.16 & 6.53 & -8 & -16 & -5 & 7 & 1071 & 0.09 & 5.28 & -6 & -28 & -9 \\
\hline Cerebellar vermis & 7 & 2070 & 0.28 & 8.13 & 0 & -48 & -9 & 7 & 2191 & 0.06 & 6.12 & 2 & -44 & -12 \\
\hline Cerebellum I & 9 & 1784 & 0.39 & 6.00 & -29 & -59 & -25 & 9 & 2157 & 0.23 & 5.89 & -37 & -54 & -29 \\
\hline $\begin{array}{l}\text { Cerebellum r } \\
p<0.001\end{array}$ & 9 & 3018 & 0.23 & 8.39 & 17 & -40 & -24 & 10 & 2538 & 0.09 & 5.52 & 18 & -38 & -23 \\
\hline
\end{tabular}

ROIs are listed. Activations correlated with TCS and ICS are characterized by the number of subjects showing the activation $(n), \mathrm{BOLD}$ contrast, and $t$ value $(t)$. Coordinates in Talairach space are given $(x, y, z)$. The sides that are not listed showed no activation. I, Left; $r$, right.

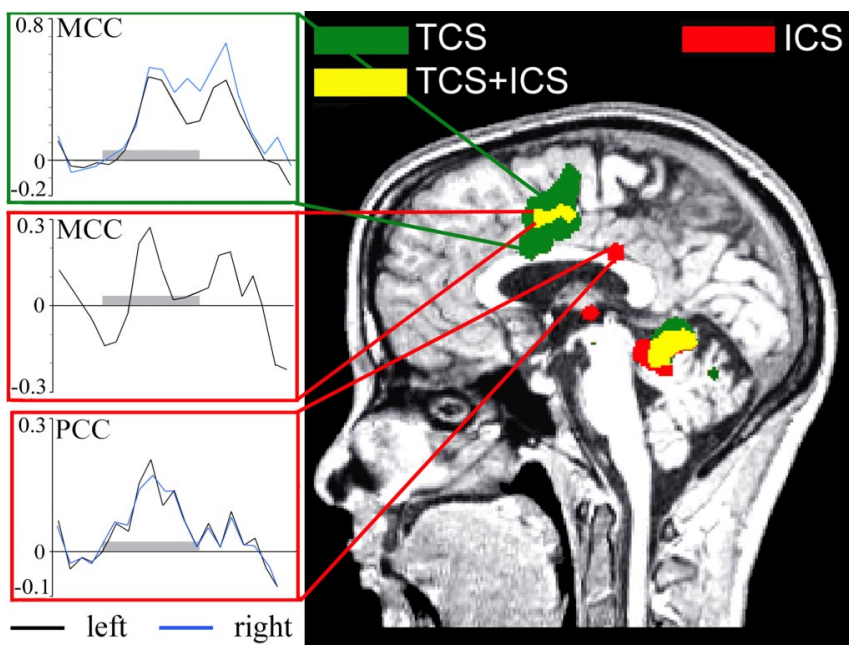

Figure 3. Multi Studies of activations by both stimuli in a standardized Talairach brain. Activation by ICS is plotted in red; activation by TCS is plotted in green. Common activation by ICS and TCS is shown in yellow. This sagittal slice shows activation in the cingulate cortex, thalamus, and cerebellar vermis. Only ICS activates the PCC, whereas TCS dominates in the MCC. The mean relative changes of the BOLD signals in the MCC and PCC during the stimuli are plotted on the left: the time courses over the five stimulation periods are averaged for all subjects. They include $15 \mathrm{~s}$ before the $20 \mathrm{~s}$ stimulation period and $20 \mathrm{~s}$ after stimulation. TCS (green frame) and ICS (red frame) activations are linked to the corresponding clusters. The BOLD signal of the left hemisphere is plotted in black, and the BOLD signal of the right side is plotted in blue. Stimulation periods are indicated by the gray bars on the $x$-axis.

no significant differences in activation between male and female subjects.

Analysis of signal change over time revealed a second peak after stimulus termination in most brain regions. Interestingly, time courses displayed a symmetrical pattern within the ROI with bilateral activation. Different stimuli, however, evoked distinct reactions in the same ROI. Example time courses are plotted in Figures 3-5.

Although most ROIs were bilaterally activated, there were some remarkable exceptions. During ICS stimulation, only the contralateral medial cingulate cortex (MCC) [Brodmann's area
(BA) 24] and medial (BA 13) and posterior (BA 14, 15, 16) insular cortex and brainstem were activated. TCS lead to an exclusive contralateral activation in the posterior insular cortex (PIC) and brainstem.

Most ROIs were responsive to both types of stimulation. Generally, TCS resulted in larger clusters, stronger changes in BOLD contrast, and higher $t$ values.

However, some areas were selectively or predominantly activated by one stimulus type. Table 2 lists the significant contrasts of ICS and TCS including Talairach coordinates. In cases of differently located activations by ICS and TCS, both clusters are listed.

Most notably, the SMA (BA 6) was activated only by TCS, whereas the posterior cingulate cortex (PCC) (BA 23, 29, 30, 31) was only activated by ICS (Fig. 3). Contrast analysis emphasizes the finding in the PCC and the left SMA. TCS activation in the right SMA does not produce a significant contrast to ICS. Some other areas, in particular the medial insular cortex (MIC) and MCC, showed ipsilateral activation only during TCS (Fig. 4). However, contrast analysis could only verify a significant dominance of TCS in the MIC. Figure 3 shows selective activation in the PCC by ICS and activation in the MCC.

Although Multi Studies analysis does not imply major differences in S2, left PIC, cerebellar vermis (vermis), and left cerebellar hemisphere (Fig. 4, 5), contrast analysis reveals a significant dominance of TCS in these regions (Table 2).

Even when both types of stimuli excited the same ROI, the activated fields were not always identical, particularly in the thalamus (Fig. 4) and brainstem (Fig. 5). For these areas, contrast analysis reveals significant clusters with slightly different coordinates for both stimuli.

\section{Discussion}

This study differentiates parts of the cerebral network of pain that are responsible for input from cutaneous afferents including or excluding mechano-insensitive C-nociceptors. To separate $\mathrm{CM}_{\mathrm{i}}$, we used ICS to excite cutaneous nerve endings including $\mathrm{CM}_{\mathrm{i}}$, as evidenced by the axon-reflex flare (Fig. 2). In contrast, TCS recruited no or only a negligible amount of $\mathrm{CM}_{\mathrm{i}}$, as evidenced by 
Table 2. Contrast evaluation of brain ROls

\begin{tabular}{|c|c|c|c|c|c|c|c|c|c|c|}
\hline \multirow[b]{2}{*}{ Region } & \multicolumn{5}{|l|}{ TCS } & \multicolumn{5}{|l|}{ ICS } \\
\hline & $x$ & $y$ & $z$ & $t$ & $p$ & $x$ & $y$ & $z$ & $t$ & $p$ \\
\hline S2I & -52 & -30 & 24 & 3.46 & 0.0005 & & & & & \\
\hline$S 2 r$ & 52 & -28 & 16 & 2.78 & 0.0055 & & & & & \\
\hline SMAI & -2 & -3 & 49 & 2.54 & 0.0112 & & & & & \\
\hline $\mathrm{MICr}$ & 39 & -6 & 14 & 2.31 & 0.0212 & & & & & \\
\hline PICI & -35 & -19 & 8 & 2.59 & 0.0096 & & & & & \\
\hline $\mathrm{PCCl}$ & & & & & & -5 & -28 & 29 & -2.55 & 0.0109 \\
\hline $\mathrm{PCC} r$ & & & & & & 4 & -25 & 29 & -2.54 & 0.0112 \\
\hline Thalamus I & -4 & -4 & 3 & 2.73 & 0.0065 & -4 & -25 & 5 & -2.50 & 0.0126 \\
\hline Thalamus r & 2 & -11 & -5 & 2.30 & 0.0217 & 2 & -19 & 5 & -1.95 & 0.0498 \\
\hline Brainstem I & -7 & -26 & -28 & 3.53 & 0.0004 & -3 & -30 & -11 & -2.35 & 0.0189 \\
\hline Brainstem r & 7 & -19 & -25 & 2.80 & 0.0051 & & & & & \\
\hline Cerebellar vermis & -1 & -48 & -2 & 3.87 & 0.0001 & & & & & \\
\hline Cerebellum I & -30 & -39 & -28 & 3.12 & 0.0018 & -47 & -53 & -30 & -3.65 & 0.0003 \\
\hline $\begin{array}{l}\text { Cerebellum r } \\
n<005\end{array}$ & 20 & -56 & -26 & 3.17 & 0.0016 & & & & & \\
\hline
\end{tabular}

ROIs with significant difference between TCS and ICS are listed. Activations are characterized by the corresponding $t$ and $p$ values. Talairach coordinates $(x, y, z)$ denotes the center of gravity of the clusters. The sides that are not listed showed no activation. The left part of the table lists those regions that had a significantly higher BOLD contrast during TCS compared with ICS. The right part of the table lists regions with the reverse effects. In case of multiple activations within the same brain region but with different coordinates of TCS and ICS, both are listed. Note that the contrast was TCS versus ICS. Therefore, $t$ values are negative when the BOLD changes were higher during ICS than during TCS. I, Left; $r$, right.
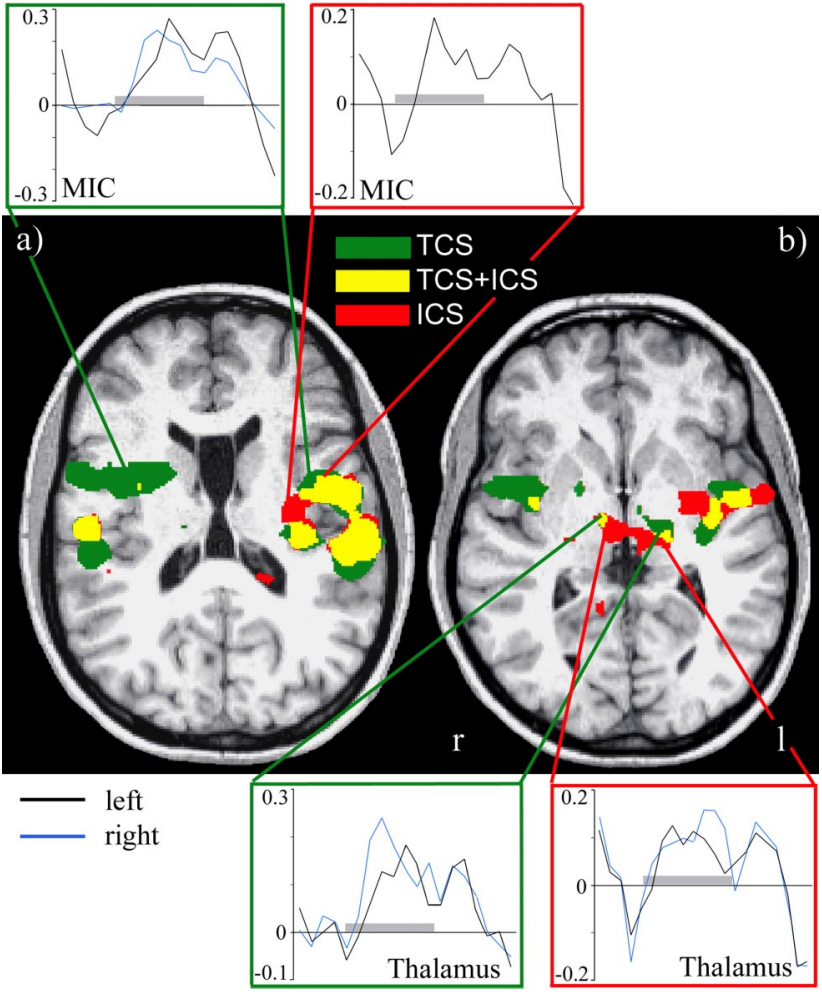

Figure 4. MultiStudies as in Figure 3. Activation by ICS and by TCS isin red and green, respectively, and common activation is in yellow. BOLD signals of the left hemisphere are plotted on the right side and are contralateral to the stimulus. $\boldsymbol{a}$, This axial slice shows that insular activation by ICS is strictly contralateral, whereas TCS activates the MIC in both hemispheres. S2 activation is found bilaterally during both types of stimulation. $\boldsymbol{b}$, This axial slice shows bilateral thalamus activation in both stimuli. Thalamus activation by TCS activation is rostral to ICS activation. Activation over time is plotted for the medial and PIC $(\boldsymbol{a})$ and the thalamus $(\boldsymbol{b})$. The time courses over the five stimulation periods are averaged for all subjects. They include $15 \mathrm{~s}$ before the $20 \mathrm{~s}$ stimulation period and $20 \mathrm{~s}$ after stimulation. TCS (green frame) and ICS (red frame) activations are linked to the corresponding clusters. The BOLD signal of the lefthemisphere is plotted in black, and the BOLD signal of the right side is plotted in blue. Stimulation periods are indicated by the gray bars on the $x$-axis.

the absence of an axon reflex. These stimulation paradigms have implications that deserve further comment. The voltage of TCS had to be higher to achieve equal pain intensities. Therefore, TCS induced a greater current spread and recruited more cutaneous
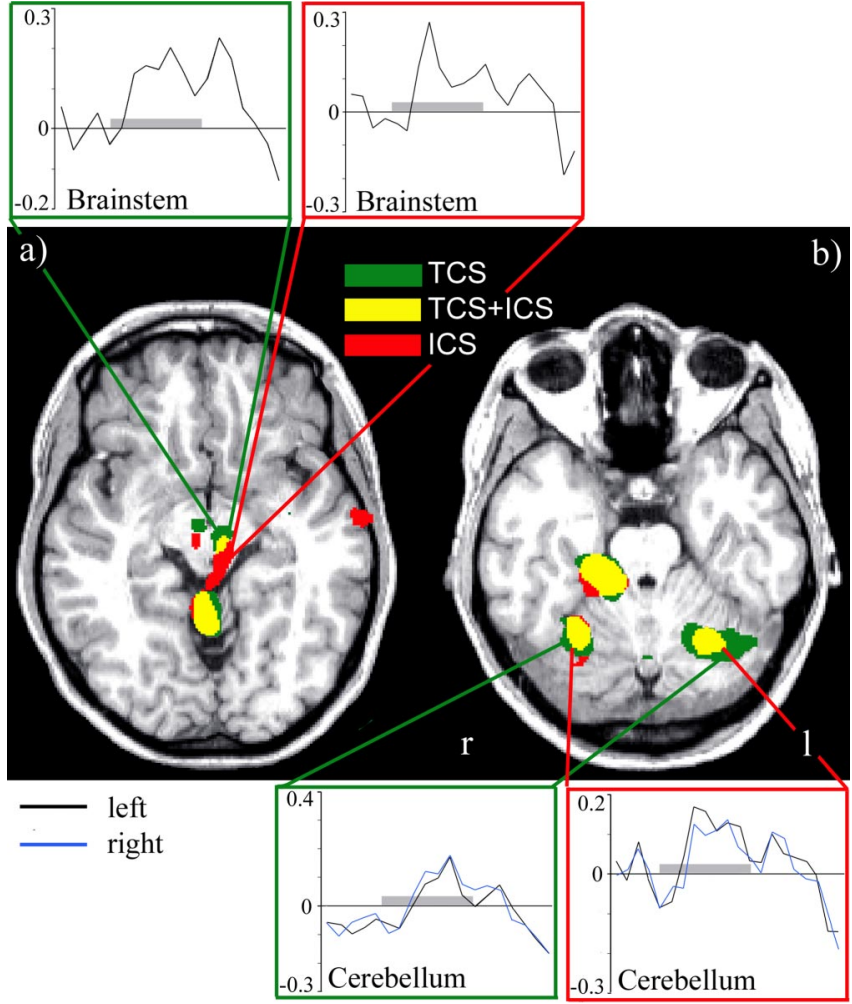

Figure 5. Multi Studies as in Figure 3. Activation by ICS and TCS is in red and green, respectively, and common activation is in yellow. $\boldsymbol{a}$, This axial slice shows activation in the contralateral brainstem and the cerebellar vermis. $\boldsymbol{b}$, This axial slice shows bilateral activation in the $A C$ during both types of stimulation. Activation over time in is plotted for brainstem $(\boldsymbol{a})$ and cerebellum $(\boldsymbol{b})$. The time courses over the five stimulation periods are averaged for all subjects. They include $15 \mathrm{~s}$ before the $20 \mathrm{~s}$ stimulation period and $20 \mathrm{~s}$ after stimulation. TCS (green frame) and ICS (red frame) activations are linked to the corresponding clusters. The BOLD signal of the left hemisphere is plotted in black, and the BOLD signal of the right side is plotted in blue. Stimulation periods are indicated by the gray bars on the $x$-axis.

units including $\mathrm{A} \delta, \mathrm{CMH}$, and other afferents. Low-threshold muscle afferents were probably not recruited because, at the dorsum of the foot, no superficial musculature is close by. In comparison, ICS with its high current density recruited the same type of afferents plus $\mathrm{CM}_{\mathrm{i}}$. This is known from studies on primary afferents and evidenced by the axon reflex. Our group has studied 
the afferent and efferent innervation of the lower leg and foot with microneurography for years (Torebjörk et al., 1996). Among hundreds of C-fibers recorded, no low-threshold C-mechanoreceptors (Olausson et al., 2002) have been encountered in this region. They are probably characteristic for the hairy skin in other parts of the limbs. C-cold units are rare compared with nociceptors (Serra et al., 1999) and have electrical thresholds that are lower or in the same range as $\mathrm{CMH}$. Although $\mathrm{A} \delta$ units were not as systematically studied as C-fibers, their electrical thresholds were always lower than those of $\mathrm{CM}_{\mathrm{i}}$ and usually also lower than those of $\mathrm{CMH}$ (our unpublished observations). It cannot entirely be excluded that unknown types of A $\delta$ units exist and are recruited by ICS but not by TCS. Studies in the monkey have shown that some A $\delta$ units have both myelinated and unmyelinated terminal branches (Peng et al., 1999). However, the thickest of these branches have the lowest electrical thresholds and are recruited by TCS. Even if unknown fiber types exist, our stimulus paradigms differentiate between a group of nociceptors responsible for the axon reflex flare versus those that induce pain but no axon reflex flare. When brain regions were preferentially activated by TCS, it was certainly caused by the quantitatively greater input. However, preferential activations by ICS must be attributed to the qualitative (presumably $\mathrm{CM}_{\mathrm{i}}$ input) and not the quantitative differences.

We differentiated three parts of the cerebral network of pain responsible for the following: both painful stimuli (ICS plus TCS), preferential input from $\mathrm{CMH}$ and $\mathrm{A} \delta$ (TCS), and input from $\mathrm{CM}_{\mathrm{i}}$ (applying subtraction ICS minus TCS). Besides a common network in both stimuli, we found that mainly input from TCS activates regions involved in nocifensive motor reactions, whereas ICS activates PCC, suggesting a role in processing $\mathrm{CM}_{\mathrm{i}}$ input.

\section{Common cerebral network of pain}

The common network comprises S2 (BA 43), insular cortex, MCC, thalamus, brainstem, vermis, and anterior cerebellar hemisphere (AC). These areas belong to the most consistently activated regions in pain imaging studies along with S1, perigenuate cingulate cortex, and anterior insula (Peyron et al., 2001; Helmchen et al., 2003; Apkarian et al., 2005). These three regions, however, were not included in our measurements. Our focus lay on insula, cingulate, and thalamus in addition to subcortical areas including the cerebellum and brainstem. Zambreanu et al. (2005) recently pointed out their eminent role in pain perception.

We found contralateral brainstem activation during both stimuli. Given the proximity to subthalamic nuclei and substantia nigra, this activation might be a correlate of motor inhibition because subjects had to depress nocifensive movements. It might also reflect ascending pathways to the thalamus because TCS activation is located rostral to ICS in the brainstem and thalamus. However, an exact allocation to specific tracts or nuclei cannot be made for anatomical variation.

\section{Pain processing network activated by TCS}

Motor thalamic nuclei, SMA, and ipsilateral MIC are selectively activated and S2, MCC, vermis, and ipsilateral AC are predominantly activated by TCS. They apparently contribute to a pain processing loop involved in the nocifensive motor response.

We found overlapping TCS and ICS activation in the posterior ventral and lateral thalamic nuclei. These nuclei manage sensory integration and project to S1, S2, and the parietal association cortex. TCS additionally activated the anterior and lateral ventral nuclei with integrative motor function and prominent projections to the primary motor cortex, premotor cortex, and SMA.

Both stimuli activated bilateral S2 that is involved in sensory discrimination and integration. Contrast analysis revealed a significant dominance of TCS that may be attributed to spatial encoding of the exact pain location to plan withdrawal.

The insula is known as a central station of pain processing important in visceral (Augustine et al., 1996), sympathetic (Sandroni et al., 1998), emotional (Morris, 2002; Kong et al., 2006), memory (Treede et al. 2000), and discriminative (Coghill et al., 1999; Peyron et al., 1999; Hua et al., 2005) aspects of pain perception. We found two foci of insular cortex activation: MIC and PIC (Figs. 4, 5). Both stimuli evoked contralateral MIC and PIC activation. This might reflect the memory and emotional component of pain or account for sympathetic activation. However, TCS dominated in the contralateral PIC that accounts for discrimination (Brooks et al., 2005). Activation threshold differences between the ipsilateral and contralateral MIC suggest that pain (Mailhofner et al., 2004; Peyron et al., 2004) or even pain intensity (Coghill et al., 2001) is encoded in this region. Although likewise painful, TCS evoked bilateral MIC activation, whereas ICS was strictly contralateral. This suggests a role of MIC beyond pain intensity encoding. Considering the functional differences of both stimuli (acute vs inflammatory pain) and the different cortical activation patterns, we suggest that MIC is involved in the setup of withdrawal.

The MIC has multiple connections with areas involved in pain perception (Jasmin et al., 2004). Besides S2 and the thalamus (Craig et al., 1994), MIC directly interacts with MCC (BA 24) (Friedman and Murray, 1986; Friedman et al., 1986). In concert with the insular response, we found bilateral MCC activation in TCS, whereas ICS only activated the contralateral hemisphere. However, contrast analysis revealed no significant activation for TCS over ICS. The MCC is involved in motor nocifensive and autonomic reactions (Bromm, 2001), skeleto-motor orientation, and fear avoidance (Vogt, 2005). Morecraft et al. (1992) found BA 23c and 24c (MCC) projections to the SMA in the rhesus monkey that suggest a functional circuit.

The SMA is only active during TCS. Previous studies have shown SMA activation by noxious heat (Coghill et al., 1994; Becerra et al., 1999; Chen et al., 2002) and capsaicin (Iadarola et al., 1998). Coghill et al. (1994) suggested a role of the SMA in movement planning and readiness to withdraw.

Helmchen et al. (2003) have postulated a specific role in pain perception for regions in the anterior cerebellum and the vermis. We found vermis activation in both stimuli. This is consistent with previous pain imaging studies (Casey et al., 1994, 1996; Becerra et al., 1999; Brooks et al., 2002) and probably caused by direct spinocerebellar input (Ekerot et al., 1991; Iadarola et al., 1998). Consistent with previous reports (Coghill et al., 2001; Bingel et al., 2002), both stimuli evoked bilateral AC activation. Contrast analysis revealed significant dominance of TCS in the vermis and ipsilateral AC, a possible correlate of motor integration (Saab and Willis, 2003) and conditioning of nocifensive responses (Ploghaus et al., 1999, 2000).

The interconnections of S2, MIC, motor thalamic nuclei, MCC, and SMA combined with the coincidence of activation during TCS and the dominance of TCS in the cerebellum suggest that these regions comprise a pain processing loop. According to the main functions of the contributing regions, this loop is involved in the coordination of motor nocifensive behavior. Only TCS evoked this activation pattern, elicited by recruitment of a larger number of cutaneous afferents. 


\section{Pain processing network activated by ICS}

Interestingly, only ICS activated the PCC. We suggest that this is as a result of the additional input from $\mathrm{CM}_{\mathrm{i}}$. According to Vogt et al. (2003), 84\% of cingulate activation during noxious heat is found in the MCC. This inconsistent activation of the PCC (Becerra et al., 1999; Chen et al., 2002; Valet et al., 2004) by thermal stimulation may be attributed to the higher heat thresholds of $\mathrm{CM}_{\mathrm{i}}$ compared with $\mathrm{CMH}$ (Weidner et al., 1999). The PCC seems to play an important role in the processing of $\mathrm{CM}_{\mathrm{i}}$ input and thereby inflammation pain and hyperalgesia.

In conclusion, our stimulation paradigms excite different nociceptor subclasses that are primarily processed in brain regions well known to be involved in pain perception. However, there are important differences between ICS and TCS. Regions involved in nocifensive motor reactions are preferentially activated by TCS. In contrast, only ICS activated PCC, generally thought to be involved in pain memory and aversion (Bromm, 2001) and emotional processes (Vogt, 2005). Our data suggest a role of PCC in processing $\mathrm{CM}_{\mathrm{i}}$ input and provide insight into the cerebral integration of hyperalgesia and inflammatory pain.

\section{References}

Apkarian AV, Bushnell MC, Treede RD, Zubieta JK (2005) Human brain mechanisms of pain perception and regulation in health and disease. Eur J Pain 9:463-484.

Augustine JR (1996) Circuitry and functional aspects of the insular lobe in primates including humans. Brain Res Brain Res Rev 22:229-244.

Becerra LR, Breiter HC, Stojanovic M, Fishman S, Edwards A, Comite AR, Gonzalez RG, Borsook D (1999) Human brain activation under controlled thermal stimulation and habituation to noxious heat: an fMRI study. Magn Reson Med 41:1044-1057.

Bingel U, Quante M, Knab R, Bromm B, Weiller C, Buchel C (2002) Subcortical structures involved in pain processing: evidence from single-trial fMRI. Pain 99:313-321.

Boynton GM, Engel SA, Glover GH, Heeger DJ (1996) Linear systems analysis of functional magnetic resonance imaging in human V1. J Neurosci 16:4207-4221.

Bromm B (2001) Brain images of pain. News Physiol Sci 16:244-249.

Brooks JC, Nurmikko TJ, Bimson WE, Singh KD, Roberts N (2002) fMRI of thermal pain: effects of stimulus laterality and attention. NeuroImage 15:293-301.

Brooks JC, Zambreanu L, Godinez A, Craig AD, Tracey I (2005) Somatotopic organisation of the human insula to painful heat studied with high resolution functional imaging. NeuroImage 27:201-209.

Casey KL, Minoshima S, Berger KL, Koeppe RA, Morrow TJ, Frey KA (1994) Positron emission tomographic analysis of cerebral structures activated specifically by repetitive noxious heat stimuli. J Neurophysiol 71:802-807.

Casey KL, Minoshima S, Morrow TJ, Koeppe RA (1996) Comparison of human cerebral activation pattern during cutaneous warmth, heat pain, and deep cold pain. J Neurophysiol 76:571-581.

Chen AC, Niddam DM, Crawford HJ, Oostenveld R, Arendt-Nielsen L (2002) Spatial summation of pain processing in the human brain as assessed by cerebral event related potentials. Neurosci Lett 328:190-194.

Coghill RC, Talbot JD, Evans AC, Meyer E, Gjedde A, Bushnell MC, Duncan GH (1994) Distributed processing of pain and vibration by the human brain. J Neurosci 14:4095-4108.

Coghill RC, Sang CN, Maisog JH, Iadarola MJ (1999) Pain intensity processing within the human brain: a bilateral, distributed mechanism. J Neurophysiol 82:1934-1943.

Coghill RC, Gilron I, Iadarola MJ (2001) Hemispheric lateralization of somatosensory processing. J Neurophysiol 85:2602-2612.

Craig AD, Bushnell MC, Zhang ET, Blomqvist A (1994) A thalamic nucleus specific for pain and temperature sensation. Nature 372:770-773.

Damasio H (1995) Human brain anatomy in computerizes images. New York: Oxford UP.

Ekerot CF, Garwicz M, Schouenborg J (1991) Topography and nociceptive receptive fields of climbing fibres projecting to the cerebellar anterior lobe in the cat. J Physiol (Lond) 441:257-274.
Friedman DP, Murray EA (1986) Thalamic connectivity of the second somatosensory area and neighboring somatosensory fields of the lateral sulcus of the macaque. J Comp Neurol 252:348-373.

Friedman DP, Murray EA, O’Neill JB, Mishkin M (1986) Cortical connections of the somatosensory fields of the lateral sulcus of macaques: evidence for a corticolimbic pathway for touch. J Comp Neurol 252:323-347.

Garell PC, McGillis SL, Greenspan JD (1996) Mechanical response properties of nociceptors innervating feline hairy skin. J Neurophysiol 75:1177-1189.

Gasser HS (1935) Conduction in nerves in relation to fiber types. Res Publ Assoc Res Nerv Med Dis 15:35-59.

Helmchen C, Mohr C, Erdmann C, Petersen D, Nitschke MF (2003) Differential cerebellar activation related to perceived pain intensity during noxious thermal stimulation in humans: a functional magnetic resonance imaging study. Neurosci Lett 335:202-206.

Hua LH, Strigo IA, Baxter LC, Johnson SC, Craig AD (2005) Anteroposterior somatotopy of innocuous cooling activation focus in human dorsal posterior insular cortex. Am J Physiol Regul Integr Comp Physiol 289:R319-R325.

Iadarola MJ, Berman KF, Zeffiro TA, Byas-Smith MG, Gracely RH, Max MB, Bennett GJ (1998) Neural activation during acute capsaicin-evoked pain and allodynia assessed with PET. Brain 121:931-947.

Jasmin L, Burkey AR, Granato A, Ohara PT (2004) Rostral agranular insular cortex and pain areas of the central nervous system: a tract-tracing study in the rat. J Comp Neurol 468:425-440.

Kong J, White NS, Kwong KK, Vangel MG, Rosman IS, Gracely RH, Gollub RL (2006) Using fMRI to dissociate sensory encoding from cognitive evaluation of heat pain intensity. Hum Brain Mapp, in press.

Kretschmann H-J, Weinrich W (1992) Cranial neuroimaging and clinical neuroanatomy. New York: Thieme Medical Publishers.

Lynn B, Schutterle S, Pierau FK (1996) The vasodilator component of neurogenic inflammation is caused by a special subclass of heat-sensitive nociceptors in the skin of the pig. J Physiol (Lond) 494:587-593.

Maihofner C, Handwerker HO (2005) Differential coding of hyperalgesia in the human brain: a functional MRI study. NeuroImage 28:996-1006.

Maihofner C, Schmelz M, Forster C, Neundorfer B, Handwerker HO (2004) Neural activation during experimental allodynia: a functional magnetic resonance imaging study. Eur J Neurosci 19:3211-3218.

Meyer RA, Campbell JN (1981) Evidence for two distinct classes of unmyelinated nociceptive afferents in monkey. Brain Res 224:149-152.

Morecraft RJ, Van Hoesen GW (1992) Cingulate input to the primary and supplementary motor cortices in the rhesus monkey: evidence for somatotopy in areas 24c and 23c. J Comp Neurol 322:471-489.

Morris JS (2002) How do you feel. Trends Cogn Sci 8:317-319.

Olausson H, Lamarre Y, Backlund H, Morin C, Wallin BG, Starck G, Ekholm S, Strigo I, Worsley K, Vallbo AB, Bushnell MC (2002) Unmyelinated tactile afferents signal touch and project to insular cortex. Nat Neurosci 9:900-904.

Peng YB, Ringkamp M, Campbell JN, Meyer RA (1999) Electrophysiological assessment of the cutaneous arborization of Adelta-fiber nociceptors. J Neurophysiol 82:1164-1177.

Peyron R, Garcia-Larrea L, Gregoire MC, Costes N, Convers P, Lavenne F, Mauguiere F, Michel D, Laurent B (1999) Haemodynamic brain responses to acute pain in humans: sensory and attentional networks. Brain 122:1765-1780.

Peyron R, Laurent B, Garcia-Larrea L (2001) Functional imaging of brain responses to pain. A review and meta-analysis (2000). Neurophysiol Clin 30:263-288.

Peyron R, Schneider F, Faillenot I, Convers P, Barral FG, Garcia-Larrea L, Laurent B (2004) An fMRI study of cortical representation of mechanical allodynia in patients with neuropathic pain. Neurology 63:1838-1846.

Ploghaus A, Tracey I, Gati JS, Clare S, Menon RS, Matthews PM, Rawlins JNP (1999) Dissociating pain from its anticipation in the human brain. Science 284:1979-1981.

Ploghaus A, Tracey I, Clare S, Gati JS, Rawlins JN, Matthews PM (2000) Learning about pain: the neural substrate of the prediction error for aversive events. Proc Natl Acad Sci USA 97:9281-9286.

Ringler R, Greiner M, Kohlloeffel L, Handwerker HO, Forster C (2003) BOLD effects in different areas of the cerebral cortex during painful mechanical stimulation. Pain 105:445-453. 
Saab CY, Willis WD (2003) The cerebellum: organization, functions and its role in nociception. Brain Res Brain Res Rev 42:85-95.

Sandroni P (1998) Testing the autonomic nervous system. Int Assoc Study Pain Newsletter, November.

Schaible HG, Schmidt RF (1988) Excitation and sensitization of fine articular afferents from cat's knee joint by prostaglandin E2. J Physiol (Lond) 403:91-104.

Schmelz M, Schmidt R, Ringkamp M, Handwerker HO, Torebjörk HE (1994) Sensitization of insensitive branches of $C$ nociceptors in human skin. J Physiol (Lond) 480:389-394.

Schmelz M, Schmidt R, Bickel A, Handwerker HO, Torebjörk HE (1997) Specific C-receptors for itch in human skin. J Neurosci 17:8003-8008.

Schmelz M, Michael K, Weidner C, Schmidt R, Torebjörk HE, Handwerker HO (2000) Which nerve fibers mediate the axon reflex flare in human skin? NeuroReport 11:645-648.

Schmidt R, Schmelz M, Forster C, Ringkamp M, Torebjörk E, Handwerker H (1995) Novel classes of responsive and unresponsive C nociceptors in human skin. J Neurosci 15:333-341.

Schmidt R, Schmelz M, Torebjörk HE, Handwerker HO (2000) Mechanoinsensitive nociceptors encode pain evoked by tonic pressure to human skin. Neuroscience 98:793-800.

Schmidt R, Schmelz M, Weidner C, Handwerker HO, Torebjörk HE (2002) Innervation territories of mechano-insensitive $\mathrm{C}$ nociceptors in human skin. J Neurophysiol 88:1859-1866.

Serra J, Campero M, Ochoa J, Bostock H (1999) Activity-dependent slowing of conduction differentiates functional subtypes of $\mathrm{C}$ fibres innervating human skin. J Physiol (Lond) 515:799-811.
Talairach J, Tournoux P (1988) Co-planar stereotaxic atlas of the human brain, 3-dimensional proportional system: an approach to cerebral imaging. New York: Thieme Medical Publishers.

Torebjörk HE, Schmelz M, Handwerker HO (1996) Functional properties of human cutaneous nociceptors and their role in pain and hyperalgesia. In: Neurobiology of nociceptors (Belmonte C, Cervero, F, eds), pp 349369. New York: Oxford UP.

Treede RD, Apkarian AV, Bromm B, Greenspan JD, Lenz FA (2000) Cortical representation of pain: functional characterization of nociceptive areas near the lateral sulcus. Pain 87:113-119.

Valet M, Sprenger T, Boecker H, Willoch F, Rummeny E, Conrad B, Erhard P, Tolle TR (2004) Distraction modulates connectivity of the cingulofrontal cortex and the midbrain during pain-an fMRI analysis. Pain 109:399-408.

Vogt BA (2005) Pain and emotion interactions in subregions of the cingulate gyrus. Nat Rev Neurosci 6:533-544.

Vogt BA, Berger GR, Derbyshire SW (2003) Structural and functional dichotomy of human midcingulate cortex. Eur J Neurosci 18:3134-3144.

Weidner C, Schmelz M, Schmidt R, Hansson B, Handwerker HO, Torebjörk HE (1999) Functional attributes discriminating mechano-insensitive and mechano-responsive $\mathrm{C}$ nociceptors in human skin. J Neurosci 19:10184-10190.

Zambreanu L, Wise RG, Brooks JC, Iannetti GD, Tracey I (2005) A role for the brainstem in central sensitisation in humans. Evidence from functional magnetic resonance imaging. Pain 114:397-407. 